\title{
Discussion
}

This study sheds a welcome light on the issue of the dental on the children with intellectual disability. The goal of the study was to focus on the discrepancies between the ethical values of the dentists, their principles and their daily practices when treating children with disability. The starting point for discussion was a clinical scenario designed to facilitate a discussion of approaches to care and then to lead into ethical reflection.

The results of this study are discussed following the thematic analysis and in relation to data from the literature. Minor themes are also explored and the emergent ethical issues discussed.

\section{First Contact}

According to the literature, it seems preferable for the dentist to fetch the patient from the waiting room personally, as this shows involvement with the patient (confidence building relationship). ${ }^{14}$ However, the dental assistant can also be considered as playing a true role in dental care, passing on valuable information to practitioners.

Concerning behaving differently with a child with intellectual disability, opinions were divided. How is the patients' status considered, how are they seen by the dentists? Does the practitioner only look at them from the angle of their difference and their disability? The framework of inclusion demands that each patient has the right to be treated as an "ordinary" patient. We have to consider the patients first as a person, with all their differences, and we must focus on their individuality in order to provide attention and appropriate care. ${ }^{22,25}$ However, the term "intellectual disability" refers to a social construct. When a dental session is described as "care performed in a child with intellectual disability", a change in attitude may be unintentionally introduced ${ }^{26}$. 
The terms and phrases used to introduce the consultation were not the same for all practitioners. But for all dentists questioned here, attitudes follow the principles of beneficence. Introducing the dental session by talking about something else creates a social relationship with the patient and possibly with the parents. It places the child in a context which is not limited to treating a "mouth" and intellectual disability, or even a more restricted field such as the "teeth". This technique, known as distraction, is approved by many authors on the grounds that it has the advantage of inducing a positive state of mind in the patient, who was initially anxious because of the stressful situation. ${ }^{27}$

\section{Information}

All the practitioners interviewed provided the child with information about the session ahead. Some practitioners used other media as a complement to convey information. Communication is the key to the behavioural approach, but the content of the message delivered is also important. ${ }^{28} \mathrm{~A}$ recent study explored the role of caregivers in decisionmaking in special care dentistry. ${ }^{29}$ This inquiry has highlighted that caregivers were highly sensitive to the place that the dentist leaves to the patient first and then to the caregiver. The recognition of the place of each by the others seems essential in order to establish a relation of care of quality.

Both professional and non-professional caregivers have been shown to be essential elements in relaying information to the patient and in assisting the patient to express his or her point of view. ${ }^{30}$ The concern of the caregivers towards the patients pushed them toward to decrease the imbalance between the various interveners: dentist and patient, and more particulary in the case of children with intellectual disability. The role of caregivers in the collection of both consent and assent deserves further attention. 
The tell-show-do strategy is widely used in paediatrics. It has been shown to be effective as long as the child has the cognitive ability to assimilate the knowledge that what they have been shown and what they have touched will be used to treat them. ${ }^{31}$ Foreshadowing and visualisation are similar concepts that use positive images and carefully chosen words to explain to a patient what to expect during treatment procedures. ${ }^{32}$ Tell-show-do provides an additional benefit because it also has an educational role in building the trust of parents and patients. $^{33}$

Practitioners cited the cuddly toy as an information carrier. This technique, known as modelling, can be effective in patients with some types of intellectual disabilities, ${ }^{34}$ but is not suited to all patients. ${ }^{35}$ One study demonstrated that desensitisation was more effective than modelling for people with intellectual disabilities. ${ }^{36}$ Information therefore needs to be provided in better ways and although some authors have proposed solutions, ${ }^{37,38}$ there is no consensus.

Another group of practitioners preferred diverting the patient's attention. They did not want to "make the patient anxious by giving too many details." Certain patients with intellectual disability have a short attention span and may be remarkably sensitive to distraction techniques. Use of such techniques may allow the patient to focus their mind on another subject while treatment is being given. ${ }^{38} \mathrm{~A}$ barrier still remains however, between the patient and the practitioner, as the mode of communication does not allow for understanding between the two.

Two trends emerged from the interviews: some practitioners try as best they can reach an agreement with the patient in distress ("even if we do not always succeed"), while others give up, discouraged by the magnitude of the task. This situation is paradoxical. Children with mental disabilities are more anxious because understanding the context and the dental care situation is difficult. They need appropriate information, provided with tools adapted to their 
disability. The way to inform our patients has to be adapted to their receptivity. An effort has to be made to connect with the patient and to communicate information adequately. ${ }^{39}$ The question of information raises the issue of informed consent in these children. In France, since 2002, the emergence of patient rights in the French healthcare system has totally changed the doctor patient relationship. Patients should become actors in health decisions that concern them, and the practitioner should play the role of informant and counsellor. In the case of children with intellectual disabilities the question of informed consent is complicated. The principles of inclusion should allow this population to take advantage of the benefits of the same health activities as those experienced by people without disabilities.

\section{Attitude toward the patient}

Reflection by practitioners on the dental treatment care in a person with an intellectual disability is part of an ethical approach. The consequences of forced treatment can be dramatic. ${ }^{40}$ Providing dental care despite the patient's tears causes mixed feelings for the practitioner. Because the practitioners wish to be beneficent, it is not desirable to give care under restraint. However, it is difficult for a professional to accept that differing dental treatment or providing "no treatment" may sometimes be an adequate solution. How were the practitioners able to assess refusal? Whether they used standardised scales or asked the parents, all practitioners wished to know why the child refused to open their mouth. Professionals may tend to minimise the psychic life of patients with intellectual disability and, by extension, minimise the pain and anxiety of vulnerable patients who cannot express their feelings verbally. In a stressful situation, practitioners are eager to end the treatment session and will sometimes choose the "quick" solution, without taking the time to re-evaluate pain or anxiety when the patient begins to stir or protest. Evaluation of anxiety and pain should be an ongoing process throughout treatment. Local anaesthesia to treat pain and cognitive behavioural approaches, supplemented or not by conscious sedation, can help to resolve an 
apparent refusal of care. Agitation is an indicator of discomfort and time must be taken to remedy it.

Once, or if, the hypothesis of pain can be eliminated, the dentist faced with refusal of care from a child with intellectual disabilities must make a choice. Will he/she sacrifice the autonomy of the patient or the principle of beneficence? The ethical debate appears here when the solution is not obvious. What arguments will help her make his/her decision (fig 1)? If the priority for him/her is to eliminate pain and infection, thus protecting the vulnerable person, he/she will probably favour the principle of beneficence. However, if the consent of the patient, informed consent and adequate information are arguments which take precedence, he/she will rather promote the principle of autonomy.

\section{Outcome from the practitioner's viewpoint}

A large majority of practitioners interviewed were satisfied with the session, even though it ended with restraint of the patient (fig 2). Others raised the issue of the possible consequences of forced treatment on the patient: withdrawal, agitation and injury in the short term, and refusal of the next treatment session in the long term. The principle of non-maleficence thus conflicts with the principle of beneficence. Is the patient's physical and mental integrity respected when it takes "three or four to maintain him"? Does an attitude of "No matter what it costs" may, in some situations, represent unreasonable obstinacy?

Satisfaction or dissatisfaction with the outcome of the consultation are the two major divergent influences on decision-making when practitioners are faced with a moral dilemma. According to Le $\mathrm{Coz}^{41}$, two argument systems are available to solve our moral problems in clinical care: deontology and utilitarianism. In the first model, the fundamental marker is the duty to respect autonomy and equal dignity. Everyone must be treated as similar (equal) carrying an absolute value (dignity). In the utilitarian model, the fundamental reference is the natural desire for happiness. The goal is to seek happiness for the greatest number, identifying 
the individual's interest with the universal interest. So, if the practitioner in question adopts a utilitarian argument, restraint of the agitated patient is a necessary evil and is less important than the service rendered: "the tooth was treated", "the patient is no longer in pain", "the parents are reassured." The practitioner may therefore feel "satisfied" with the session. However, if the practitioner in question adopts a deontological argument, nothing can justify having forced the patient to have "benefited" from their own vulnerability.

This study approached the question of dental treatment in children with intellectual disabilities from the standpoint of the dentist. The question now needs to be addressed from the viewpoint of the person with intellectual disability. Their own subjective position is neglected in these reflections and it is essential to introduce the notion of reciprocity into the question. In the field of disability studies, the patient must participate directly to enrich the debate $^{42}$. In the case of children with intellectual disability, access to reciprocity will also undoubtedly imply the active participation of caregivers.

\section{Limitations of the study}

The results of this study need to be considered within its inherent limits. As for all qualitative data, the results cannot be generalised. The number of participants was small and non-representative. Moreover, all the dentists worked within the same region and within the same network. However, trends emerged and two main profiles arose quickly. These profiles allowed reflection as to how professionals reason when faced with discrepancies between values and clinical practice. Interpretation of the results is also limited by the study design. All the interviews, transcription, coding and analysis were undertaken by one person. This was a positive point, in that the researcher was able to fully appropriate the data. However, it led to the risk of subjective interpretation, which would have been avoided if several investigators had been involved. Also, audio recording allows for greater accuracy of 
transcription than contemporaneous researcher notes and reliability would have been improved if participants had been given the opportunity to check their own transcript for accuracy ${ }^{43}$. During development of the protocol however, a conscious decision was taken not to record the interviews to avoid the participants feeling that they were being judged as to their professional ability during the discussion.

\section{Conclusion}

Intellectual disability raises the question of human values and the meaning of physical and intellectual autonomy. In this context, ethical reflection is necessary to avoid detachment from the patient or, worse, neglect or abuse through use of excessive restraint during treatment. Work with vulnerable people implies taking risks and assessing the complex relationships established between individuals and patients, relatives and caregivers. Ethical reflection may help to consider and to clarify different alternatives. The challenge to the health care professional is far greater than that of simply achieving beneficence; it is also that of how best to inform vulnerable patients (e.g. by finding appropriate and accessible tools) to reach real agreement and therefore obtain better cooperation in the chair. The work presented here has clarified the different perspectives of dentists, who for the majority were shown to adopt a utilitarian point of view. Further study into the experiences of the patients themselves and their accompanying caregivers could greatly enrich this reflection. Improved media to convey information and the development of more effective behavioural guidance techniques to improve quality of assent are necessary. 